\title{
Experiments in Automated Lexicon Building for Text Searching
}

\author{
Barry Schiffman and Kathleen R. McKeown \\ Department of Computer Science \\ Columbia University \\ New York, NY 10027, USA \\ $\{$ bschiff,kathy\}@cs.columbia.edu
}

\begin{abstract}
This paper describes experiments in the automatic construction of lexicons that would be useful in scarching large document collections for text fragments that address a specific information need, such as an answer to a question.
\end{abstract}

\section{Introduction}

In developing a system to find answers in toxt to user questions, we uncovered a major obstacle: Document sentences that contained answers did not often use the same expressions as the culestion. While answers in documents and questions use terms that are related to each other, a system that scarches for answers based on the question wording will often fail. 'To address this problen, we devoloped techniques to antomatically build a lexicon of associated terms that can bo used to help find appropriate toxt segments.

The mismatch between question and document wording was brought home to us in an analysis of a tostbod of question/answer pairs. Wo had a collection of newswire articles about the Clinton impeachment to use as a small-scale corpus for devolopment of a system. We asked several people to pose cuestions about this woll-known topic, but we did not make the corpus available to our contributors. We wanted to avoid questions that tracked the terminology in the corpus too closely to simulate questions to a real-world system. 'The result was a set of cuuestions that used language that rarely matched the pluasing in the corpus. We had expected that we would be able to make most of these lexical connections with the help of Wordnct (Miller, 1990).

For example, consider a simple question about testimony: "Did Secret Service agents give testimony about Bill Clinton?" There is no ronson to expect that the answer would appear baldly stated as "Secret Service agents did testify ..." What we need to know is what testimony is about, where it occurs, who gives it. I'he answer would be likely to be found in a passage mentioning juries, or prosecutors, like these found in our Clinton corpus:

Starr immodiately brought Sccret Service cmployees before the grand jury for questioning.

Prosecutors repeatedly asked Secret Service persomel to repeat gossip they may have heard.

Yet, the Wordnet synsets for "testimony" offer: "evidence, assertion, averment and asseveration," not a very holpful selection here. Wordnet hypernyms become general quickly: "declaration," "indication" and "information" are only one stop up in the hicrarchy. Following these does not lead us into a courtroom.

We asked our contributors for a second round of questions, but this time made the corpus available to them, explaining that we wanted to be sure the answers were contained in the collection of articles. 'Tlio result was a set of fuestions that much more closely matched the wording in the corpus. 'J'his was, in fact, what the 1999 DARPA question-answering competition did in order to ensure that their questions could be answered (Singhal, 1999). 'The second question-answering conference adopted a new approach to gathering questions and verifying separately that they are answerable.

Our intuition is that if we can find the typical lexical neighborhoods of concepts, wo can efficiently locate a concept clescribed in a query or a question without noeding to know the precise way the answer is phrased and without relying on a costly, handbuilt concept hicrarchy.

The example above illustrates the point. Testimony is given by witnesses, defendants, eyowitnesses. It is solicited by prosecutors, counsols, lawyers. It is heard by judges, juries at trials, hearings, and recorded in depositions and transcripts. What we wanted was a complete description of the world of testimony - the who, what, when and where of the word. Or, in other words, the "metaaboutness" of terms.

I'o this end, wo experinnented using shallow linguistic techniques to gather and analyze word cooccurrence data in various configurations. Unlike previous collocation rescarch, we were interested in an expansive set of relationships between words 
rather than a specific relationship. More important, we felt that the information we needed could be derived from an analysis that crossed clause and sentence boundaries. We hypothesized that news articles would be cohcrent so that the sequences of sentences and clauses would be linked conceptually.

We examined the nouns in a number of configurations - paragraphs, sentences, clauses and sequences of clauses - and obtained the strongest results from configurations that count co-occurrences across the surface subjects of sequences of two to six clauses. Experiments with multi-clause configurations were generally more accurate in a variety of experiments.

In the noxt section, we briefly review related research. In section 3 we describe our experiments. In section 4, we discuss the problem of evaluation, and look ahead to future directions in the concluding sections.

\section{Related Work}

There has been a large body of work in the collection of co-occurrence data from a broad spectrum of perspectives, from information retrieval to the development of statistical methods for investigating word similarity and classification. Our efforts fall somewhere in the middle.

Compared with document retrieval tasks, we are more closely focused on the words themselves and on specific concepts than on document "aboutness." Jing and Croft (1994) examined words and phrases in paragraph units, and found that the association data improves retrieval performance. Callan (1994) compared paragraph units and fixed windows of text in examining passage-level retrieval.

In the question-answering context, Morton (1999) collected document co-occurrence statistics to uncover part-whole and synonymy relationships to use in a question-answering system. 'The kcy difference here was that co-occurrence was considered on a whole-document basis. Harabagiu and Maiorano (1999) argued that indexing in question answering should be based on paragraphs.

One recent approach to automatic lexicon building has used seed words to build up larger sets of semantically similar words in one or more categories (Riloff and Shepherd, 1997). In addition, Strzalkowski and Wang (1996) used a bootstrapping technique to identify types of references, and Riloff and Jones (1999) adapted bootstrapping techniques to lexicon building targeted to information extraction.

In the same vein, researchers at Brown University (Caraballo and Charniak, 1999), (Berland and Charniak, 1999), (Caraballo, 1999) and (Roark and Charniak, 1998) focused on target constructions, in particular complex noun phrases, and searched for information not only on identifying classes of nouns, but also hypernyms, noun specificity and meronymy.
We have a different perspective than these lines of inquiry. They were specifying various semantic relationships and seeking ways to collect similar pairs. We have a less restrictive focus and are rolying on surface syntactic information about clauses.

For more than a decade, a variety of statistical techniques have been developed and refined. The focus of much of this work was to develop the methods themselves. Church and Hanks (1989) explored the use of mutual information statistics in ranking co-occurrences within five-word windows. Smadja (1992) gathered co-occurrences within fiveword windows to find collocations, particularly in specific domains. Hindle (1990) classified nouns on the basis of co-occurring patterns of subjectverb and verb-object pairs. Hatzivassiloglou and McKeown (1993) clustered adjectives into semantic classes, and Pereira et al. (1993) clustered nouns on their appearance in verb-object pairs. We are trying to be less restrictive in learning multiple salient relationships between words rather than seeking a particular relationship.

In a way, our idea is the mirror image of Barzilay and Elhadad (1997), who used Wordnet to identify lexical chains that would coincide with cohesive text segments. We assumed that documents are cohesive and that co-occurrence patterns can uncover word relationships.

\section{Experiments}

The focus of our experiment was on units of text in which the constituents must fit together in order for the discourse to be colicrent. We made the assumption that the documents in our corpus were coherent and reasoned that if we had enough text, covering a broad range of topics, we could pick out domainindependent associations. For example, testimony can be about virtually anything, since anything can wind up in a court dispute. But over a large cnough collection of text, the terms that directly relate to the "who," "what" and "where" of testimony per se should appear in segments with testimony more frequently than chance.

These associations do not necessarily appear in a dictionary or thesaurus. When humans explain an unfamiliar word, they often use scenarios and analogies.

We divided the experiments in two groups: one group that looks at co-occurrences within a single unit, and another that looks at a sequence of units.

In the first group of experiments, we considered paragraphs, sentences and clauses, cach with and without prepositional phrases.

- Single paragraphs with/without PP

- Single sentences with/without PP

- Single clauses with/without PP 
In the second group, wo considered two clauses and sequences of subject noun phrases from two to six clauses. In this group, we had:

" 'Two clauses with/without pp

- A sequence of subject NPs from 2 clauses

- A sequence of subject NPs from 3 clauses

- A sequence of subject NPs from 4 clauses

- A sequence of subject NPs from 5 clauses

- A sequence of subject NPs from 6 clauses

The intuition for the sccond group is that a topic flows from one grammatical unit to another so that the salicnt nouns, particularly the surface subjects, in successive clauses should reveal the associations we are sceking.

'To illustrate the method, consider the three-clause configuration: Say that word $d_{i}$ appcars in clausen . We maintain a table of all word pairs and increment the entries for $\left(\right.$ wor $_{i}$, wor $d_{j}$ ), where wor $d_{j}$ is a subject nom in clause $_{n}$, clause $_{n+1}$, or clause $n+2$. No effort was made to resolve pronomial references, and these were skipped.

We used nouns only'because preliminary tests showed that pairings betwoen nouns seemed to stand out. We included tokens that were tagged as proper names when they also have have common moanings. For cxample, consider the Linguistic Data Consortimm at the University of Pemsylvania. Data, Consortium and University would be on the list used to build the table of matchups with other nouns, but Penmsylvania would not. We also collocted nom modifiers as well as head nouns as they can carry more information than the surface heads, sucle as "business groul", "science class" or "crime sconc."

The corpus consisted of all the general-interest articles from the New York Times newswire in 1996 in the North American News Corpus, and did not; include either sports or business news. We first removed duplicate articles. The data from 1996 was too sparse for the sequence-of-subjects configurations. 'To balance the experiments better', we added another year's worth of newswire articles, from 1995, for the sequence-of-subject configurations so that we had more than one million matchups for each configuration (Table 1).

The process is fully automatic, requiring no suporvision or training examples. The corpus was tagged with a decision-tree tagger (Schmid, 1994) and parsed with a finitc-state parser (Abney, 1996) using a specially written context-frec-grammar that focused on locating clause boundaries. The grammar also identified extended noun phrases in the subject position, verb phrases and other noun phrases and prepositional phrases. The nomns in the tagged, parsed corpus were reduced to their syntactic roots (removing plurals from nouns) with a lookup table created from Wordnet (Miller, 1990) and CELEX (1995). We porformed this last step mainly to address the sparse data problem. There were a substantial number of pairings that occurred only once. We climinated from consideration all such singletons, although it did not appear to have much effect on the overall outcome.

\begin{tabular}{|l|r|}
\hline \hline Config & Matchups \\
\hline \hline Para +pp & 6.5 million \\
\hline Sent & 1.7 million \\
\hline Sent +pp & 4 million \\
\hline 1 Clause & 1.1 million \\
\hline 1 Clause +pp & 2.8 million \\
\hline 2 Clause & 1.9 million \\
\hline 2 Clause +pp & 5 million \\
\hline Subj 2 Clause & 1.1 million* \\
\hline Subj 3 Clause & 1.6 million* \\
\hline Subj 4 Clause & 2.1 million* \\
\hline Subj 5 Clause & 2.6 \\
\hline Subillion* \\
\hline
\end{tabular}

Table 1: Number of matchups found; the "*" denotes the inclusion of 1995 data

'There were about 1.2 million paragraphs, 2.2 million sentences and 3.4 million clauses in the selected portions of the 1996 corpus. The total number of words was 57 million. 'Table 2 shows the number of distinct nol1ns.

\begin{tabular}{|l|r|r|}
\hline & All Extracted & Counts $>1$ \\
\hline No p)s & 74,500 & 44,400 \\
\hline W/Pps & 91,700 & 53,900 \\
\hline Sulpjs & 51,000 & 30,800 \\
\hline
\end{tabular}

Table 2: Distinct Nouns, 1996 Data

To score the matchups in our initial experiments, wo used the Dice Coefficient, which produces values from 0 to 1 , to measure the association between pairs of words and then produced an ordered association list from the co-occurrence table, ranked according to the scores of the entries.

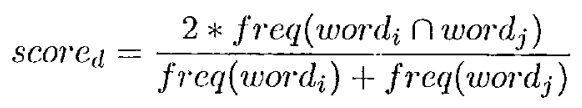

One problem was immediately apparent: The quality of the association lists varied greatly. The scoring was doing an acceptable job in ranking the words within each list, but the scores varied greatly from one list to another. Our initial strategy was to choose a cutoff, which we set at 21 for each list, and we tried several alternatives to weed out weak associations. 
In one method, we filtered the association lists by cross-referencing, removing from the association list for word $_{i}$ any word $d_{j}$ that failed to reciprocate and to give a high rank to wor $_{i}$ on its association list. Another similar approach was to try to combine evidence from different experiments by taking the results from two configurations into consideration. A third strategy was to calculate the mutual information between the target word and the other words on its association list.

$$
\operatorname{score}_{m i}=p(x y) * \log \left(\frac{p(x y)}{p(x) p(y)}\right)
$$

Using the mutual information computation provided an way of using a single measure that was able to compare matchups across lists. We set a threshold of $1 \times 10^{-6}$ for all matcliups. Thus these association lists vary in length, depending on the distributions for the words, allowing them to grow up to 40 , while some onded up with only one or two words.

\section{Evaluation}

The cvaluation of a system like ours is problematic. The judgments wo made to determine correctness were not only highly subjective but time-consuming. We had 12 large lexicons from the different configurations. We had chosen a random sample of 10 percent of the 2,700 words that occurred at least 100 times in the corpus, and manually constructed an answer key, which ended up with almost 30,000 entries.

From the resulting 270 words, we discarded 15 of those that coincided with common names of people, such as "carter," which could refer to the former American president, Chris Carter (creator of the television show "X-Files"), among others. We thought it better to delay making decisions on how to handle such cases, especially since it would require distinguishing one Carter from another. Such words presented several difficulties. Unless the individuals involved were well-known, it was often impossible to distinguish whether the system was making errors or whether the resulting descriptive terms were informative.

Tables 3 and 4 show an cxample from the answer key for the word "faculty."

The overall results from the first stage of the process, before the cross-referencing filter are shown in Table 5 , ranging from $73 \%$ to $80 \%$ correct. The configurations that included prepositional phrases and those that used sequences of subject noun plriases outperformed the configurations that relied on subjects and objects in a single grammatical unit. These differences were statistically significant, with $p<$ 0.01 in all cases.

The overall results after cross-referencing, in 'Table 6 , showed improvements of 5 to 10 percentage

\begin{tabular}{|lll|}
\hline enrollment & hiring & administrator \\
journalism & alumnus & student \\
school & union & math \\
engineering & curriculum & trustee \\
group & seminar & thesis \\
tenure & staff & department \\
mathematician & educator & member \\
ivy & arts & college \\
chancellor & report & senate \\
activism & university & chairman \\
professor & teaching & law \\
regent & doctorate & administration \\
academic & committee & sernester \\
board & campus & undergraduate \\
salary & council & research \\
president & adviser & mathematics \\
course & advisor & sociology \\
dean & study & science \\
teacher & canmon & provost \\
vote & & \\
\hline
\end{tabular}

Table 3: Answer Key for Faculty: OK

\begin{tabular}{|lll|}
\hline \hline load & traflicway & unrest \\
architecture & diversity & hurdlo \\
shicld & minority & revision \\
disburse & percent & woman \\
clement & & \\
\hline
\end{tabular}

Table 4: Answer Key for Faculty: Wrong

points, while the effect of the number of matchups was diminished. Hore, the subject-sequence configurations showed a distinct advantage. While more noise might be expected when a large segment of text is considered, these results support the notion that the underlying colherence of a discourse can be recovered with the proper solection of linguistic features. The improvements in each configuration over the corresponding configuration in the first stage were all statistically significant, with $p<0.01$. Likewise, the cdge the sequence-of-subjects configurations had over the other configurations, was also statistically significant.

The results from combining the evidence from different configurations, in Table 7 , showed a much higher accuracy, but a sharp drop in the total number of associated words found. 'I'he most fruitful pairs of experiments were those that combined distinct approaches, for example, the five-subject configuration with either full paragraphs or with sentences with prepositional phrases. It will remain unclear until we conduct a task-based evaluation whether tho smaller number of associations will be harmful.

The final experiment, computing the mutual information statistic for the matchups of a key word with co-occurring words was perhaps the most interesting because it gave us the ability to apply a 


\begin{tabular}{|llll|}
\hline Config & OK & Wrong & Pct OK \\
\hline Para +Pp & 3832 & 1054 & 78 \\
Sent & 3773 & 1270 & 75 \\
Sont +pp & 3973 & 1070 & 79 \\
1 Clause & 3652 & 1371 & 73 \\
1. Clauses +Pp & 3935 & 1108 & 78 \\
2 Clauses & 3695 & 1328 & 74 \\
2 Clauses +pp) & 3983 & 1018 & 80 \\
Subj 2 Cl & 3877 & 1139 & 77 \\
Subj 3 Cl & 3899 & 1117 & 78 \\
Subj 4 Cl & 3905 & 1082 & 78 \\
Subj 5 Cl & 3904 & 1076 & 78 \\
Subj 6 Cl & 3909 & 1066 & 79 \\
\hline
\end{tabular}

\begin{tabular}{|llll|}
\hline Config & OK & Wrong & Pct ()K \\
\hline Para & 2003 & 183 & 92 \\
Sent & 1962 & 222 & 90 \\
Sent- & 2033 & 213 & 91 \\
1. Clause & 1791 & 218 & 89 \\
1 Clauset & 2004 & 198 & 91 \\
2 Clause & 2028 & 277 & 88 \\
2 Clauset- & 2129 & 244 & 90 \\
\hline
\end{tabular}

Table 7: Results of combining evidence; all configurations were combined with the sequence of six subjects

'Table 5: Results Before Cross Referencing'

\begin{tabular}{|llll|}
\hline Config & OK & Wrong & Pet OK \\
\hline Para +pp) & 3650 & 734 & 83 \\
Sont & 3328 & 742 & 82 \\
Sont +pp & 3751 & 818 & 82 \\
1 Clause & 3067 & 748 & 80 \\
1 Clauses +pp & 3659 & 826 & 82 \\
2 Clauses & 3048 & 554 & 85 \\
2 Clauses +pl) & 3232 & 604 & 84 \\
Subj 2 Cl & 2910 & 450 & 87 \\
Subj 3 Cl & 3020 & 440 & 87 \\
Subj 4 Cl & 3050 & 428 & 88 \\
Subj 5 Cl & 3133 & 442 & 88 \\
Sulpj 6 Cl & 3237 & 149 & 88 \\
\hline
\end{tabular}

\begin{tabular}{|llll|}
\hline Config & OK & Wrong & Pet OK \\
\hline Para +pp & 4923 & 807 & 86 \\
Scnt & 5193 & 990 & 84 \\
Sent tpp) & 4876 & 775 & 86 \\
1 Clause: & 5299 & 1233 & 81 \\
1 Clauses +pp) & 5047 & 878 & 85 \\
2 Clauses & 5025 & 928 & 84 \\
2 Clauses +pp) & 4668 & 728 & 87 \\
Subj 2 Cl & 5229 & 939 & 85 \\
Subj 3 Cl & 5187 & 860 & 85 \\
Subj 4 Cl & 5119 & 808 & 86 \\
Subj 5 Cl & 5003 & 764 & 87 \\
Subj 6 Cl & 4980 & 736 & 87 \\
\hline
\end{tabular}

Table 8: Results with mutual information

'Jable 6: Results After Cross Referencing

single threshold across different licy words, saving the effort of performing the cross-referencing calculations and providing a deeper assortment in some cases. In most of the configurations, mutual information gave us more words, and greater precision at the same time, but most of all, gave us a reasonable threshold to apply throughout the experiment. While the accuracios in most of the configurations were close to one another, those that nsed only single units tended to be weaker than the multi-clauso units. Note that the paragraph configuration was tested with far more data than any of the others.

Oul system makes no effort to account for loxical ambiguity. The uses wo intend for our lexicon should provide some insulation from the effects of polysemy, since scarches will be conducted on a 11umber of terms, which should converge to one meaning. It is clear that in lists for key words with multi ple senses, the dominant sense where there is one, appeass much more frequently, such as "faculty ," where the meaning of "teacher" is more firequent than the meaning of "ability." Figure 1 shows the top 21. words in the seculunce-of-six subjects, before the cross-leferencing filter was applied. T'wenty of the 21 entiries were scored acceptable.

After the cross-reforencing is applied, doctorate, education and revision were climinated.

The results from the single clause configuration (Figure 2) were almost as strong, with three crrors, and a lair amount of overlap betwoen the two.

Jhe word "admiral" was more difficult for the experiment using the Dice coefficient. The list shows some of the confusion arising from our stratery on proper nouns. Admiral would be expected to occur with many proper namos, including some that are spelled like common noms, but the list for the single clause $t_{-p p}$ ) configuration presented a puzzling list (Figure 3).

The sparseness of the data is also apparent, but it was the dog references that appeared quite strange at a glance: Inspection of the articles showed that thoy came from an article on the pots of famous people. Note that the dogs alid not appear in top ranks of the sequence of subjects configuration in the Dice experiment (Figure 4), nor were they in the results from the experiments with cross-referencing; combining cridenco and mutual information.

After cross-referencing, the much-shorter list for the Subj-6 configuration had "aviator", "break-up", "commander", "decoration", "equal-opportunity", "flect", "merino", "navy", "pearl", "promotion", "rear", and "short".

the combined-evidence list contained only eight words: "navy", "short", "aviator", "merino", "dishonol", "decoration", "sub" and "break-up".

Using the mutual information scoring, the list in the Subj-6 configuration for admiral had only 
faculty - trustee(51) $0.053 ;$ campus(41) 0.045 ; college(1.13) 0.034; member(369) 0.028 ; professor(102) 0.028 ; university(203) 0.027 ; student(206) 0.025 ; regent (19) 0.025 ; tenure(15) 0.025 ; chancel$\operatorname{lor}(28) 0.023$; administrator(34) 0.023 ; provost(12) 0.023; dean(27) 0.021; alumnus(13) 0.021; math(12) 0.017 ; revision $(8) \quad 0.013$; salary(13) 0.013 ; sociology (7) 0.013 ; educator(11) 0.012 ; doctorate $(6)$ 0.011 ; teaching $(9) 0.011$;

Figure 1: The top-ranked matchups for "faculty" from the Subj-6-Clause configuration before cross-referencing. The numbers in parentheses are the number of matchups and the real numbers following are the scores. Errors are in bold

faculty - trustee(31) 0.033 ; member (266) 0.025 ; administrator(31) 0.023 ; colloge(42) 0.012 ; dean(15) 0.012 ; tenure (8) 0.011; ivy(6) 0.011; stafr(33) 0.01 ; semester(6) 0.01 ; regent(7) 0.01 ; salary(12) 0.01 ; $\operatorname{math}(7) 0.008$; professor(31) 0.008 ; $\operatorname{load}(6) \quad 0.007$; curriculum(5) 0.006 ; revision(4) 0.006 ; minor$\operatorname{ity}(11) 0.006$;

Figure 2: The top-ranked matchups for "faculty" under the single clause configuration. Errors are in bold.

nine words: "navy", "general", "commander", "vice", "promotion", "officer", "fleet", "military" and "smith."

Finally, the even-sparser mutual information list for the paragraph configuration lists only "navy" and "suicide."

\section{Conclusion}

Our results are encouraging. We were able to decipher a broad type of word association, and showed that our method of searching sequences of subjects outperformed the more traditional approaches in finding collocations. We believe we can use this technique to build a large-scale lexicon to holp in difficult information retrieval and information extraction tasks like question answering.

The most interesting aspect of this work lies in the system's ability to look across several clauses and strengthen the connections between associated words. We are able to deal with input that contains numerous errors from the tagging and shallow parsing processes. Local context has been studicd extensively in recent years with sophisticated statistical tools and the availability of enormous amounts of text in digital form. Perhaps we can expand this perspective to look at a window of perhaps several sentences by extracting the correct linguistic units in order to cxplore a large range of language processing problems. admiral - navy(41) 0.027 ; ayalon(4) 0.024 ; cheating(5) 0.02 ; gallantry(3) 0.016 ; chow(4) 0.015 ; serviceman(4) 0.013 ; short(3) 0.013 ; wardroom(2) 0.012 ; american(2) $0.012 ; \operatorname{cnos}(2) \quad 0.012$; solfassessment(2) $0.011 ; \quad$ merino(2) $0.011 ; \quad \operatorname{occlot}(2)$ 0.011 ; wolfhound(2) 0.011 ; igloo(2) 0.011 ; paprika(2) $0.011 ;$ spaniel(2) $0.01 ; \operatorname{medal}(8) \quad 0.01$; awc(3) 0.01; pedigrec(2) 0.009; terricr(2) 0.009;

Figure 3: Top-ranked matchups for "admiral" under the clause $+\mathrm{pp}$ configuration.

admiral - navy (88) 0.071 ; short(7) 0.03 ; promotion(11) 0.027; happiness(8) 0.026 ; fleet(11) 0.024 ; aviator(5) $0.022 ;$ ambition(8) $0.019 ;$ merino(3) 0.01 .9 ; dishonor $(3) \quad 0.018$; rear(4) 0.018 ; decoration(4) $0.015 ; \operatorname{sub}(3) \quad 0.013$; $\operatorname{airman}(3) \quad 0.013$; graveses(2) 0.012 ; submariner(2) 0.012 ; equalopportunity $(2) \quad 0.012$; break-up (2) 0.012 ; commander(18) 0.012 ; pearl(7) 0.012 ; prophecy(4) 0.012 ; torturer(2) 0.012 ;

Figure 4: The list for admiral from the Subj-6 configuration.

\section{$6 \quad$ Future Work}

- We will have the scoring key itself evaluated by people who are not involved in the research.

- We are planning to conduct task-based evajuation in question answering.

- We are considering deploying a named entity module to provide some classification of which proper nouns should be counted and which should not.

- We plan to experiment with ways to incorporate using examining verbs and making use of surface objects in the configurations with sequences of clauses, as well as strengthen the finite state grammar.

- We will explore using the system to extract biographic information.

\section{Acknowledgments}

This material is based upon work supported by the National Science Foundation under grants Nos. IIS96-19124 and IRI-96-18797, and work jointly supported by the National Science Foundation and the National Library of Medicine under grant No. IIS98-17434. Any opinions, findings, and conclusions or recommendations expressed in this material are those of the authors and do not necessarily reflect the views of the National Science Foundation. 


\section{References}

Steven Abncy. 1996. Partial parsing via finite-state cascades. In Proceedings of the ESSLLI'96 Robust Parsing Workshop.

Regina Barzilay and Michael Elhadad. 1997. Using lexical chains for text summarization. In Proceedings of the Intelligent Scalable Text Summarization Workshop. ACL.

Matthew Berland and Eugone Charniak. 1999. Finding parts in very large corpora. 'Iechnical Report 'TR CS99-02, Brown University.

James P. Callan. 1994. Passage-level evidence in document retricval. In Proceedings of the Seventeenth Annual International ACM SIGIR Conference, Dublin, Ireland. ACM.

Sharon Caraballo and Eugene Chamiak. 1999. Determining the specificity of nouns from text. In Proccedings of Conference on Empirical Methods in Natural Language Processing.

Sharon Caraballo. 1999. Automatic acquisition of a hypernym-labeled nom hierarchy from text. In Procedings of the 37th Annual Mecting of the Association for Computational Linguistics, June.

CELEX, 1995. The CELEX lexical database Dutch, English, German. Centr for Lexical Information, Max Planck Institute for Psycholinguistics, Nijmegen.

Kenneth W. Church and Patrick Hanks. 1989. Word association norms, mutual information and lexicography. In Procedings of the g7th meeting of the ACL.

Sanda M. Harabagiu and Steven J. Maiorano. 1999. Finding answers in large collections of texts: Paragraph indexing + adductive inference. In Question Answering Systems. AAAI, November.

Vasilcios Hatzivassiloglou and Kathleen R. McKeown. 1993. 'Lowards the antomatic identification of adjectival scales: Clustering adjectives according to meaning. In Proceedings of the 31st Annual Mecting of the $A C L$.

Donald Hindle. 1990. Noun classification from predicate-argumont structures. In Proceedings of the 28th Annual Meeting of the ACL.

Yufeng Jing and W. Bruce Croft. 1994. An association thesaurus for information retricval, tech. rep. no 94-17. 'lechnical report, Amherst: University of Massachusetts, Center for Intelligent Information Retricval.

G. Miller. 1990. Wordnet: An on-line lexical database. International Journal of Lexicography.

'Thomas S. Morton. 1999. Using coreference for' question answering. In Procecdings of the Workshop on Coreference and Its Applications, pages 85-89, College Park, Maryland, June. Association for Computational Ijinguistics, Association for Computation Linguistics.
Fernando Pereira, Naftali Tishby, and Lillian Lee. 1993. Distributional clustering of english words. In Procedings of the 31 st Annual Mecting of the $A C L$.

Ellen Riloff and Rosie Jones. 1999. Learning dictionaries for information extraction by multilevel bootstrapping. In Proceedings of the Sixteenth National Conference on Artificial Intelligence. AAAI.

Ellen Riloff and Jessica Shepherd. 1997. A corpusbased approach for building semantic lexicons. In Proceedings of the Second Conference on Empirical Methods in Natural Language Processing.

Brian Roark and Eugene Charniak. 1998. Nounphrasae co-occurrence statistics for semiautomatic scmantic lexicon construction. In Proccedings of the 36th Annual Meeting of the Association for Computational Linguistics and the 17th International Conference on Computation Linguistics.

Helmut Schmid. 1994. Probabilistic part-of-speech tagging using decision trecs. In Procecdings of the International Conference on New Methods in Language Processing.

Amit Singhal. 1999. Question and answer track home page. WWW.

Frank Smadja. 1992. Retrieving collocations from text: Xtract. Computational Linguistics, Special Issue.

'Lomek Stryalkowski and Jin Wang. 1996. A selflearning universal concept spotter. In Procedings of the International Conference on Computational Linguistics (Coling 1996). 